\title{
Determination of necessary geometric parameters of tripod robot workspace, taking into account zones of singularity
}

\author{
Khalapyan Sergey Yuriyevich \\ Automated and information control systems department \\ Stary Oskol Technological Institute n.a. A.A. Ugarov \\ (branch) NUST "MISIS" \\ Stary Oskol, Russia \\ cx@ hotbox.ru
}

\author{
Rybak Larisa Alexsandrovna \\ Dept. of machine building technology \\ BSTU named after V.G. Shoukhov \\ Belgorod, Russia \\ rl_bgtu@intbel.ru
}

\author{
Malyshev Dmitry Ivanovich \\ Dept. of machine building technology \\ BSTU named after V.G. Shoukhov \\ Belgorod, Russia \\ rl_bgtu@intbel.ru
}

\begin{abstract}
The article considers the problem of providing the necessary geometric parameters of the workspace of a flat robot with 3 degrees of freedom. The influence of the geometric parameters of the robot mechanism configuration (drive mechanisms rod lengths and working platform parameters) as well as the working platform angle of rotation on the dimensions of the workspace was investigated. Special provisions (singularity zones) of the mechanism should be excluded from the robot workspace. The mechanism under investigation has singularities of the second type. Increasing angle $\varphi$ that decreases the size of the workspace, but increases radius $\theta$ of the circle, on which the points of singularity are located, is shown. Thus, it is necessary to limit the swing angle range and expand the variation range of the lengths of the rods' change to ensure the necessary size of the workspace mechanism. For the given dimensions of the workspace (based on the requirements of the technological process), the task of determining the geometric parameters of the robot mechanism configuration is solved. Mathematical modeling is performed. The results of computational experiments are presented.
\end{abstract}

Keywords - tripod robot,. robot mechanism configuration, mathematical modeling, computational experiments

\section{INTRODUCTION}

The main problem with the workspace representation of parallel robots is that the limitations on the degrees of freedom are all usually coupled. Hence for robots having more than 3 d.o.f., there will be no possible graphical illustration of the robot workspace. This is not usually the case with serial robots. For example, the workspace of a 6 d.o.f. serial robot with a concurrent axis wrist may be represented by the $3 \mathrm{D}$ volume that may be reached by the center of the wrist - this illustrates the translations, and by the surface that may be reached by the extremity of the end-effector (which illustrates 2 degrees of freedom in rotation). The 3D volume depends only on the motion capability of the first three actuated joints, while the orientation uses only the last three joints. A graphical representation of the workspace of parallel robots will be possible only for 3 d.o.f robots. For robots with d.o.f. $n$ $>3$, workspace representation will be possible only if one fixes $n-3$ pose parameters. According to fixed types of parameters or to the constraint that are imposed on the parameter, let us obtain different types of workspace.

The known approach to workspace calculation was suggested by Jo [1]. Taking into consideration the constraints on the joint coordinates, he transformed the inequalities that are imposed by these constraints into equalities by introducing extra variables. Then he considered the generalized coordinates (vector $\mathbf{X}$ ), the joint coordinates (vector $\boldsymbol{\Theta}$ ) and the variables (vector $\mathbf{w}$ ) that are introduced by the transformation of the inequalities into equalities.

Let $\mathbf{q}$ be the vector that is constituted of all of these unknowns. The structure of the mechanism leads to constraint equations on the components of $\mathbf{q}$ which may be written in an implicit form as $\Phi(\mathbf{q})=0$. Let $J \Phi$ be the jacobian of the system, i.e. the matrix:

$$
J_{\phi}=\frac{\partial \Phi}{\partial q}=\left(\left(\frac{\partial \Phi}{\partial X}, \frac{\partial \Phi}{\partial \Theta}, \frac{\partial \Phi}{\partial w}\right)\right)
$$

The workspace boundary is obtained as the set of vectors $\mathbf{q}$, such that for given $\mathbf{X}$, there will not be a unique set of vectors $\boldsymbol{\Theta}, \mathbf{w}$. In other words, the rank of matrix

$$
\left(\left(\frac{\partial \Phi}{\partial \Theta}, \frac{\partial \Phi}{\partial w}\right)\right)
$$

is lower than its dimension. A numerical procedure is then used to calculate the pose of the platform where this condition is satisfied. However, let us note that Jo illustrated this approach only for the simple case of the calculation of the constant orientation workspace of a 6-UPS robot. The introduction of other constraints limiting the workspace would 
lead to a jacobian, which is so large that to render the procedure is quite difficult to manage. Adkins [2] and Haugh [3] succeeded in finding a point on the boundary and using a numerical continuation method to follow the boundary. Although this approach is general, Adkins and Haugh restrict their calculation for a constant orientation workspace, as the general problem will be very complicated. But these authors were able to predict the singularity barriers that may split the workspace into different aspects (an aspect is a maximal singularity-free component of the workspace). Instead of using a continuation method to follow the boundary, some authors have proposed formulating that point as a constrained optimization problem [4].

The purpose of the geometrical method is to determine geometrically the boundary of the robot workspace. The principle is to deduce geometrical object $W l$ from the limitations on each leg that describes all the possible locations of $\mathbf{X}$, which satisfy the leg constraints. One such object is obtained for each leg and the robot workspace is constituted of the intersection of all $\mathrm{Wl}$.

In some cases such as the Delta, calculation of the workspace may be done directly by CAD [5,6] since this calculation is equivalent to the intersection of simple $3 \mathrm{D}$ volumes.

This approach is usually restricted to $3 \mathrm{D}$ workspace and is able to deal mostly with the constraints on the joint coordinates, although we will see that joint limits and interference constraints may be taken into account in some cases.

The main interest of the geometrical approach is that it is usually very fast and accurate, and provides a minimal representation of the workspace which may be used to calculate efficiently some characteristics of the workspace, such as its volume. Its drawbacks are that it must be tailored to the considered robot, it may be difficult to take into account all constraints, and the minimal representation of the workspace may not be the most appropriate for such tasks as motion planning. A possible simplified approach is to compute only slices of the workspace, and to approximate the section of $W l$ by polygons. This approach requires a good computational geometry library that is able to execute Boolean operations: intersection, union, difference on polygons with arbitrarily large number of edges.

Numerous papers, dealing with workspace calculation, use methods based on the discretization of the pose parameters in order to determine the workspace boundary. In this discretization approach, the workspace is covered by a regular grid, either cartesian or polar, of nodes. Each node is then tested to see whether it belongs to the workspace. The boundary of the workspace is constituted of the set of valid nodes, where at least one close neighbor does not belong to the workspace. The advantage of this method is that it allows one to take into account all constraints. But this approach has many drawbacks:

- the accuracy of the boundary depends on the sampling step that is used to create the grid, the computation time grows exponentially with the sampling step so that there is a limit on the accuracy.

- problems occur when the workspace possesses voids.

- the boundary representation may involve a large number of nodes.

- the boundary is used for different operations such as determination of the workspace volume, inclusion of a trajectory in the workspace, etc. When performed on a boundary represented by a discrete set of poses, these operations are computer intensive. To avoid this drawback, Chablat [7] proposed storing the workspace representation as an octree structure that allows faster motion planning and volume calculation. Still, obtaining the structure is computer and memory intensive.

\section{DETERMINATION OF THE ROBOT-TRIPOD GEOMETRIC PARAMETERS}

Let us consider the flat mechanism of the tripod robot in the form of a 3-RPR mechanism [8], moving the fixed tripod platform along the $\mathrm{x}$ and $\mathrm{y}$ axes and rotating around the $\mathrm{z}$ axis (Fig.1).

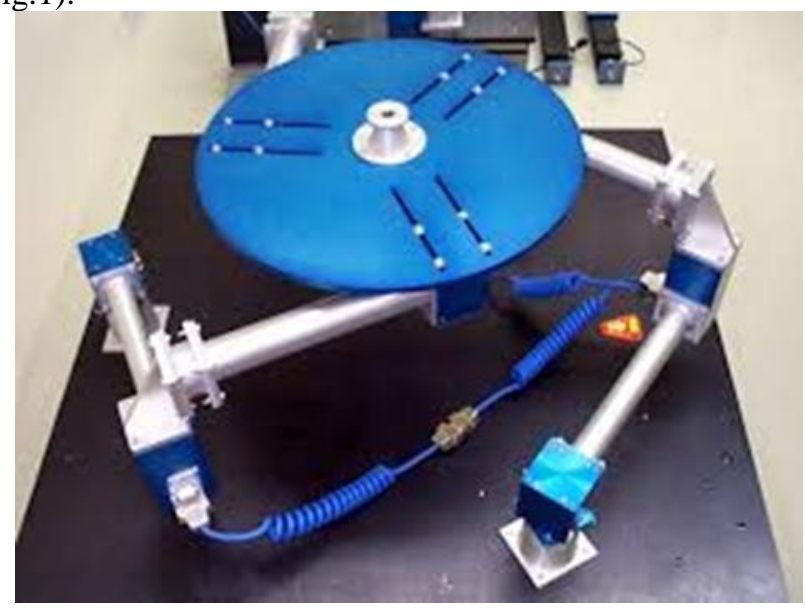

Fig. 1. Flat 3-RPR mechanism scheme

It should be noted that when the tripod moves, some parasitic movement of its movable platform along the $\mathrm{x}$ and $\mathrm{y}$ axes occurs. However, this movement can be determined by the current lengths of the drive links (or even with specified output coordinates) and easily compensated by the corresponding movement of the plane mechanism.

One of the most important subtasks of controlling the parallel structure mechanism is determination of the feedback signal by the position of the moving platform by solving the direct kinematic problem of the mechanism.

The method for solving the tripod direct kinematic problem for the purpose of controlling this mechanism was proposed by authors in [9]. It is shown that this task can be successfully solved on the basis of neural network technologies.

Thus, it is necessary to organize the solution of the direct kinematic problem for a flat 3-RPR mechanism for controlling the proposed composite mechanism with 6 degrees of freedom. Using the formula for solving the inverse problem of positions [10]: 


$$
L_{i}=\sqrt{\left(x+l_{3, i} \cos \left(\gamma_{i}+\varphi\right)-x_{A, i}\right)^{2}+\left(y-l_{3, i} \sin \left(\gamma_{i}+\varphi\right)-y_{A, i}\right)^{2}},
$$

where $(\mathrm{x}, \mathrm{y}, \varphi)$ - output mechanism coordinates, $\gamma_{\mathrm{i}}-$ angle (fig. 1), which determines the output link geometry, $\left(\mathrm{x}_{\mathrm{Ai}}, \mathrm{y}_{\mathrm{Ai}}\right)-$ coordinates of the point $A_{i}$ hinged attachment of the drive link to a stationary base, $1_{3, i}$ - distance from point $C_{i}$ of hinged attachment of the drive link to the mobile platform to the point determining the position of the output link of the mechanism, $\mathrm{L}_{\mathrm{i}}$ - length of the $\mathrm{i}$-th drive link.

In the case of constructing a flat $3-\mathrm{R} P \mathrm{R}$ mechanism in accordance with the classical scheme of the location of attachment points $A_{i}$ and $C_{i}$ at the vertices of equilateral triangles (Fig. 1), the lengths of the drive links can be determined on the basis of the following relationships:

$$
\begin{aligned}
& L_{1}=\sqrt{\left(x+r \cos \left(\frac{7 \pi}{6}+\varphi\right)-R \cos \frac{7 \pi}{6}\right)^{2}+\left(y+r \sin \left(\frac{7 \pi}{6}+\varphi\right)-R \sin \frac{7 \pi}{6}\right)^{2}}, \\
& L_{2}=\sqrt{\left(\left(x+r \cos \left(\frac{11 \pi}{6}+\varphi\right)-R \cos \frac{11 \pi}{6}\right)^{2}+\left(y+r \sin \left(\frac{11 \pi}{6}+\varphi\right)-\right.\right.} \\
& \left.\left.-R \sin \frac{11 \pi}{6}\right)^{2}\right)^{1 / 2},
\end{aligned}
$$

$L_{3}=\sqrt{\left(x+r \cos \left(\frac{\pi}{2}+\varphi\right)-R \cos \frac{\pi}{2}\right)^{2}+\left(y+r \sin \left(\frac{\pi}{2}+\varphi\right)-R \sin \frac{\pi}{2}\right)^{2}}$,

where $\mathrm{R}$ and $\mathrm{r}$ - circles circumscribed about triangles $\mathrm{A}_{1} \mathrm{~A}_{2} \mathrm{~A}_{3}$ and $\mathrm{C}_{1} \mathrm{C}_{2} \mathrm{C}_{3}$ radii, $\gamma_{1}=7 \pi / 6, \quad \gamma_{2}=11 \pi / 6, \quad \gamma_{3}=\pi / 2$, which simplifies the expression for $\mathrm{L}_{\mathrm{i}}$ :

$$
\begin{aligned}
& L_{1}=\sqrt{\begin{array}{l}
\left(x+\frac{r}{2}(\sin \varphi-\sqrt{3} \cos \varphi)+\frac{\sqrt{3}}{2} R\right)^{2}+\left(y-\frac{r}{2}(\sqrt{3} \sin \varphi+\right. \\
\left.+\cos \varphi)+\frac{R}{2}\right)^{2},
\end{array}} \\
& L_{2}=\sqrt{\begin{array}{l}
\left(x+\frac{r}{2}(\sin \varphi+\sqrt{3} \cos \varphi)-\frac{\sqrt{3}}{2} R\right)^{2}+\left(y+\frac{r}{2}(\sqrt{3} \sin \varphi-\right. \\
\left.-\cos \varphi)+\frac{R}{2}\right)^{2},
\end{array}} \\
& L_{3}=\sqrt{(x-r \sin \varphi)^{2}+(y+r \cos \varphi-R)^{2},}
\end{aligned}
$$

The workspace of the mechanism is determined by the values of $R, r$ and the range of the lengths of the drive links and can be determined using the equations given above. It is also necessary to exclude special provisions (points of singularity) of the mechanism from the working space. The 3RPR mechanism has singularities of the second type [8] for which $\operatorname{det}\left(\mathrm{J}_{\mathrm{A}}\right)=0$ :

$$
J_{A}=\left(\begin{array}{ccc}
\frac{\partial F_{1}}{\partial x} & \frac{\partial F_{1}}{\partial y} & \frac{\partial F_{1}}{\partial \varphi} \\
\frac{\partial F_{2}}{\partial x} & \frac{\partial F_{2}}{\partial y} & \frac{\partial F_{2}}{\partial \varphi} \\
\frac{\partial F_{3}}{\partial x} & \frac{\partial F_{3}}{\partial y} & \frac{\partial F_{3}}{\partial \varphi}
\end{array}\right),
$$

where

$$
\frac{\partial F_{i}}{\partial x}=2 x-2 x_{A i}+2 l_{3, i} \cos \left(\varphi+\gamma_{i}\right)
$$

$$
\frac{\partial F_{i}}{\partial y}=2 y-2 y_{A i}+2 l_{3, i} \sin \left(\varphi+\gamma_{i}\right),
$$

$$
\begin{aligned}
\frac{\partial F_{i}}{\partial \varphi}= & 2 l_{3, i}\left(y-y_{A i}+l_{3, i} \sin \left(\varphi+\gamma_{i}\right)\right) \cos \left(\varphi+\gamma_{i}\right)-2 l_{3, i}(x- \\
& \left.-x_{A i}+l_{3, i} \cos \left(\varphi+\gamma_{i}\right)\right) \sin \left(\varphi+\gamma_{i}\right),
\end{aligned}
$$

which in the case under consideration means

$$
\begin{gathered}
\frac{\partial F_{1}}{\partial x}=2 x+r(\sin \varphi-\sqrt{3} \cos \varphi)+R \sqrt{3}, \\
\frac{\partial F_{2}}{\partial x}=2 x+r(\sin \varphi+\sqrt{3} \cos \varphi)-R \sqrt{3}, \\
\frac{\partial F_{3}}{\partial x}=2 x-2 r \sin \varphi, \\
\frac{\partial F_{1}}{\partial y}=2 y-r(\sqrt{3} \sin \varphi+\cos \varphi)+R, \\
\frac{\partial F_{2}}{\partial y}=2 y+r(\sqrt{3} \sin \varphi-\cos \varphi)+R, \\
\frac{\partial F_{3}}{\partial y}=2 y+2 r \cos \varphi-2 R,
\end{gathered}
$$

$\frac{\partial F_{1}}{\partial \varphi}=\frac{r}{2}((2 y-r(\sqrt{3} \sin \varphi+\cos \varphi)+R)(\sin \varphi-\sqrt{3} \cos \varphi)+$ $+(2 x+r(\sin \varphi-\sqrt{3} \cos \varphi)+R \sqrt{3})(\sqrt{3} \sin \varphi+\cos \varphi))=$ $=\frac{r}{2}((2 y+R)(\sin \varphi-\sqrt{3} \cos \varphi)+(2 x+R \sqrt{3})(\sqrt{3} \sin \varphi+$ $\cos \varphi))=r(x(\sqrt{3} \sin \varphi+\cos \varphi)+y(\sin \varphi-\sqrt{3} \cos \varphi)+$ $+2 R \sin \varphi)$,

$\frac{\partial F_{2}}{\partial \varphi}=\frac{r}{2}((2 y+r(\sqrt{3} \sin \varphi-\cos \varphi)+R)(\sin \varphi+\sqrt{3} \cos \varphi)-$

$-(2 x+r(\sin \varphi+\sqrt{3} \cos \varphi)-R \sqrt{3})(\sqrt{3} \sin \varphi-\cos \varphi))=$ $=\frac{r}{2}((2 y+R)(\sin \varphi+\sqrt{3} \cos \varphi)-(2 x-R \sqrt{3})(\sqrt{3} \sin \varphi-$ $-\cos \varphi))=r(x(\sqrt{3} \sin \varphi+\cos \varphi)+y(\sin \varphi+\sqrt{3} \cos \varphi)+$ $+2 R \sin \varphi)$,

$$
\begin{aligned}
& \frac{\partial F_{3}}{\partial \varphi}=-2 r((y+r \cos \varphi-R) \sin \varphi+(x-r \sin \varphi) \cos \varphi)= \\
& =2 r((R-y) \sin \varphi-x \cos \varphi) .
\end{aligned}
$$

To define the working space and special positions of the 3RPR mechanism, let us set its geometric parameters: $\mathrm{R}=100$ $\mathrm{mm}, \mathrm{r}=50 \mathrm{~mm}, \mathrm{~L}_{1,2,3} \in[10 \mathrm{~mm}, 130 \mathrm{~mm}]$ and use the MatLAB package. The areas of possible changes in the linear output coordinates of the mechanism for different angles of the moving platform rotation are shown in Fig. 2. 

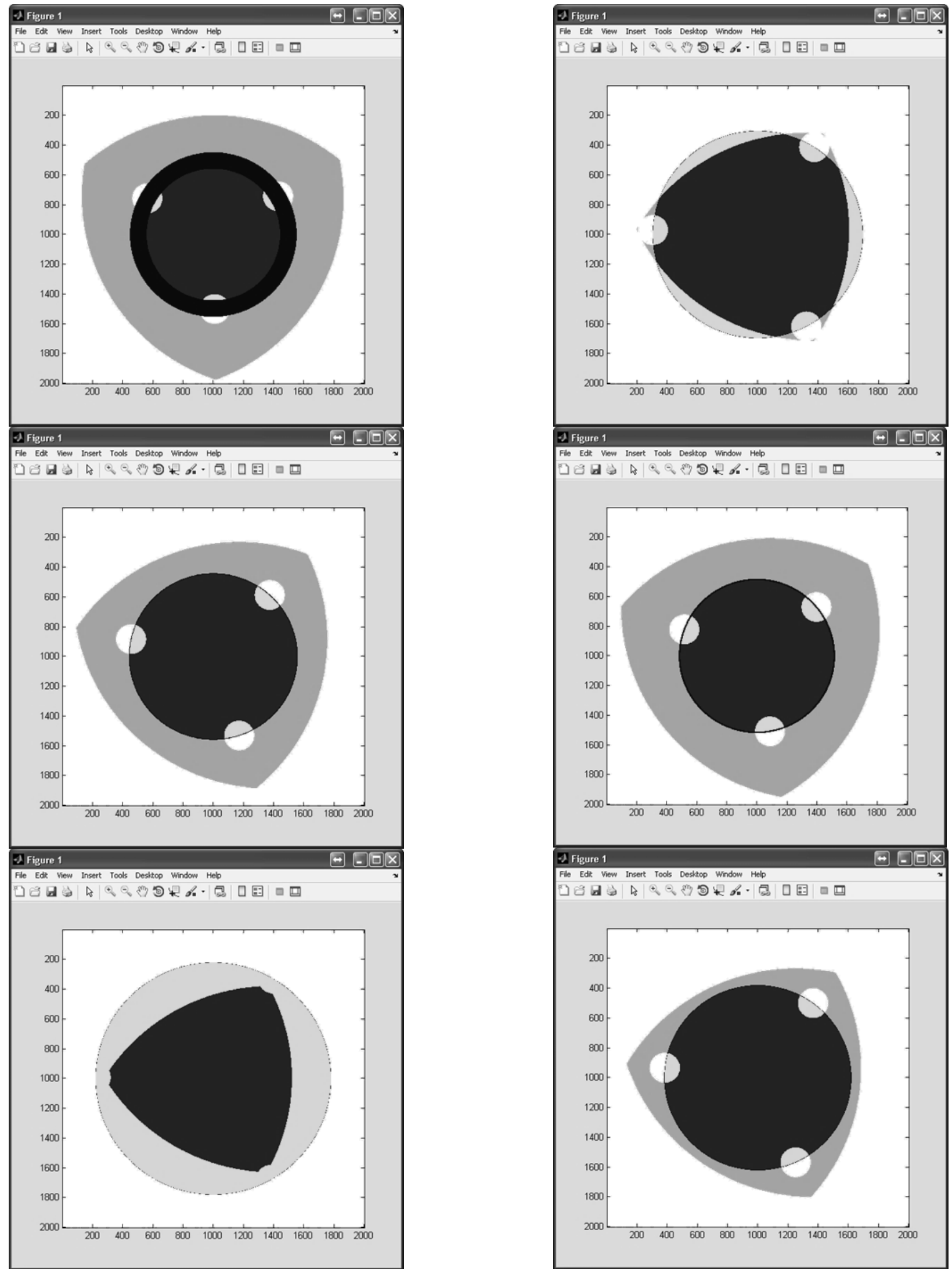

Fig. 2. The range of admissible values $\mathrm{x}$ and $\mathrm{y}$ for $\varphi=1^{\circ}, 10^{\circ}, 20^{\circ}, \ldots, 50^{\circ}$ 
Fig. 2 shows that increasing angle $\varphi$ decreases the size of the workspace, but increases radius $\theta$ of the circle on which the point of singularity is located. The dependence of radius on $\varphi$ can be determined by the cosine theorem (Fig. 3.):

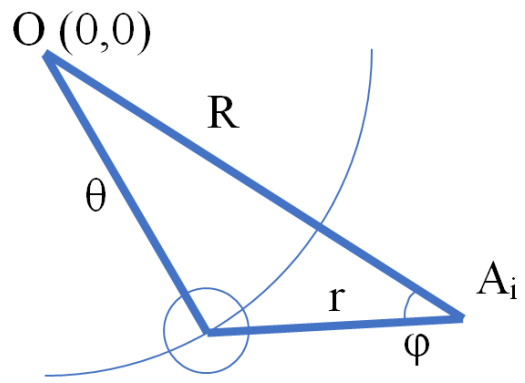

Fig. 3. Dependence of radius $\theta$ on $\mathrm{R}, \mathrm{r}$ and $\varphi$

$$
\theta(\varphi)=\sqrt{R^{2}+r^{2}-2 R r \cos \varphi}
$$

Minimum $\theta(\varphi)$ is reached when $\varphi \rightarrow 0$ :

$$
\lim _{\varphi \rightarrow 0} \theta(\varphi)=\sqrt{R^{2}+r^{2}-2 R r \cos \varphi}=|R-r|
$$

It should be noted that when $\varphi=0^{\circ}$, all points of the plane are singular. This explains Fig. 4, which shows that three straight $A_{1} C_{1}, A_{2} C_{2}$ and $A_{3} C_{3}$ intersect in a single point - the homothetic center that takes point $\mathrm{A}_{\mathrm{i}}$ at point $\mathrm{C}_{\mathrm{i}}$. The situation is similar at $\varphi=180^{\circ}$, but in this case segments $\mathrm{A}_{1} \mathrm{C}_{1}, \mathrm{~A}_{2} \mathrm{C}_{2}$ and $\mathrm{A}_{3} \mathrm{C}_{3}$ intersect, which makes rotation of the movable platform technically unrealizable.

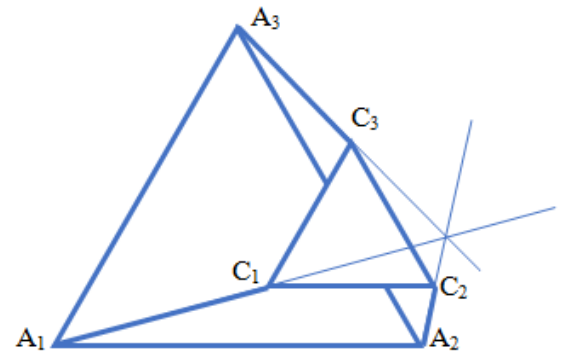

Fig. 4. The singular plane at $\varphi=0$

Thus is necessary to limit the swing angle range of $0^{\circ}<\varphi<180^{\circ}$ and to increase it to acceptable level value $\inf _{\varphi \in(0, \pi)} \theta(\varphi)=|R-r|$ and expand the range of change $\mathrm{L}_{1,2,3}$ to ensure the necessary size of the workspace mechanism, which ensures consistency of Jacobian det sign $\operatorname{det}\left(\mathrm{J}_{\mathrm{A}}\right)$. Reducing its lower limit leads to a size reduction of the "dead zones" that do not enter the workspace (white circles in Figure 2). An increase in the upper limit of the range allows increasing the size of the workspace for values of $\varphi$ close to $180^{\circ}$.

Let us note that angles above $120^{\circ}$ for the mentioned above geometric parameters of the mechanism are unattainable. It is necessary to choose new parameters that provide the movement of the movable platform center within the circle with the center at the origin and radius of $60 \mathrm{~mm}$ for the angles of rotation from $1^{\circ}$ to $179^{\circ}$. For example, a mechanism with the following geometric parameters: $\mathrm{R}=100 \mathrm{~mm}, \mathrm{r}=25$ $\mathrm{mm}, \mathrm{L}_{1,2,3} \in[10 \mathrm{~mm}, 190 \mathrm{~mm}]$ has a workspace, shown in Figure 5.
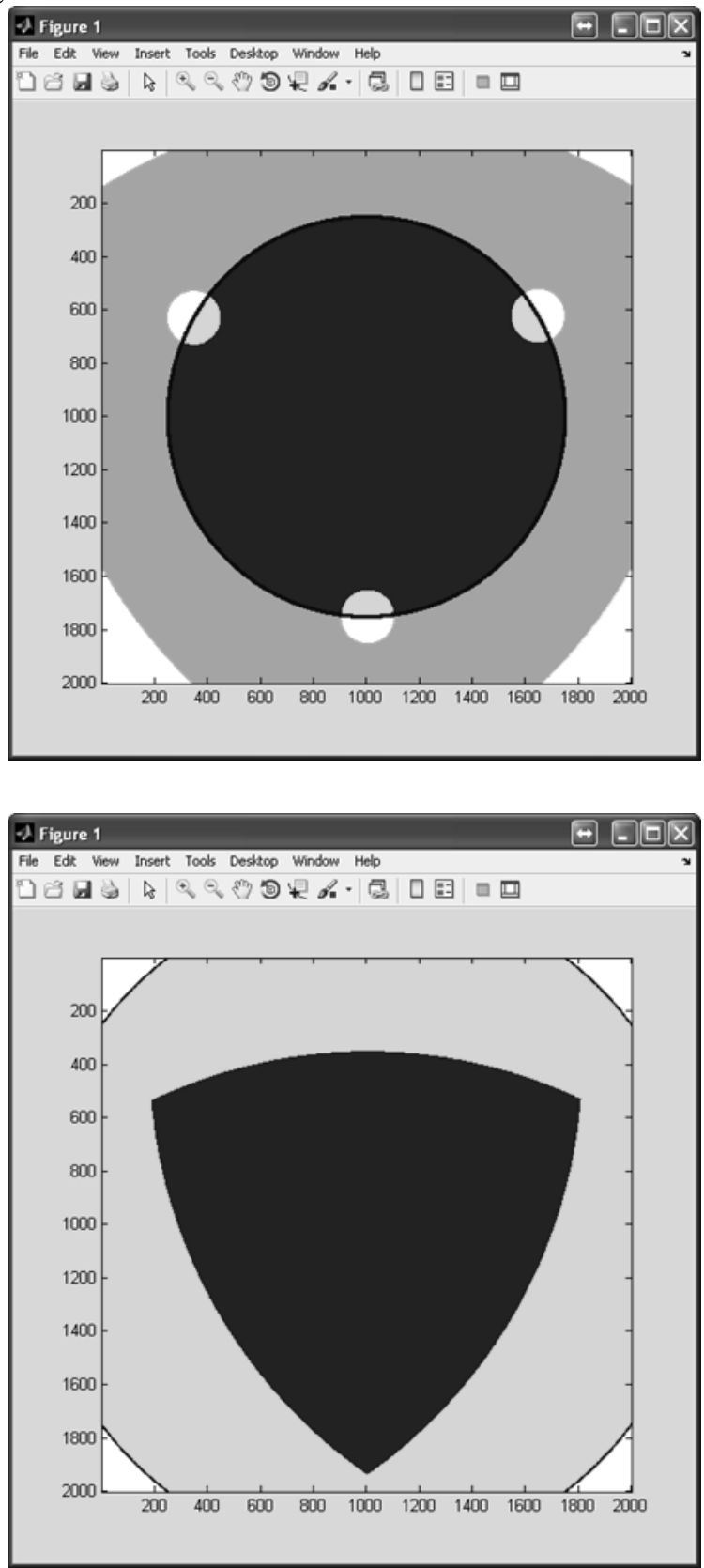

Fig. 5. Area of admissible values of $\mathrm{x}$ and $\mathrm{y}$ for mechanism with the selected parameters at $\varphi=1^{\circ}$ and $179^{\circ}$

\section{CONCLUSIONS}

An attempt to repeat the computational experiments [9] aimed at constructing the neuronet model of the mechanism showed the applicability of this methodology to the organization of an automated solution of the direct kinematic problem with the purpose of determining the feedback signal from the moving platform position. The control of the mechanism is facilitated by the absence of singularity areas in its workspace. 


\section{Acknowledgments}

The research is supported by the Russian Science Foundation, agreement number 16-19-00148

\section{References}

[1] D.Y. Jo and E.J. Haug, "Workspace analysis of closed loop mechanisms with unilateral constraints," ASME Design Automation Conf., pp. 53-60, Montreal, September 1989 [ASME Design Automation Conf., 1989].

[2] F.A. Adkins and E.J. Haug, Operational envelope of a spatial Stewart platform. ASME J. of Mechanical Design, vol. 119(2), pp. 330-332, June 1997.

[3] E.J. Haugh, F.A. Adkins, and C.M. Luh, "Operational envelopes for working bodies of mechanisms and manipulators," ASME J. of Mechanical Design, vol. 120(1), pp. 84-91, March 1998.

[4] J.A. Snyman, L.J. Du Plessis, and J. Duffy, "An optimisation approach to the determination of the boundaries of manipulator workspaces," ASME J. of Mechanical Design, vol. 122(4), pp. 447456, December 2000.
[5] R. Clavel, Conception d'un robot parall'ele rapide 'a 4 degr'es de libert'e. Ph.D. Thesis, EPFL, Lausanne, 1991, p. 925.

[6] M.L. Husty, "On the workspace of planar three-legged platforms," World Automation Congress, volume 3, pp. 339-344, Montpellier, May, 1996 [World Automation Congress, 1996].

[7] D. Chablat and P. Wenger, "Moveability and collision analysis for fullyparallel manipulators," 12th RoManSy, pp. 61-68, Paris, July, 1998 [12th RoManSy, 1998].

[8] K.G. Erastova, P.A. Laryushkin, "Research of the working area and special positions of the flat 3-RPR manipulator in the MATLAB environment," The engineering bulletin. Electronic scientific and technical journal of Bauman MSTU, №7, pp. 1-7, 2016.

[9] S.Y. Khalapyan, L.A. Rybak, A.I. Glushchenko, Y.A. Mamaev, "On neural network model development to solve parallel robots kinematics and control problems," International Journal of Pharmacy \& Technology, Vol. 8, № 4, pp. 25085-25095, 2016.

[10] P.A. Laryushkin, D.S. Epanchintseva, "The coupling equations and the solution of the inverse positional problem for plane mechanisms of a parallel structure of arbitrary geometry," The engineering bulletin. Electronic scientific and technical journal of Bauman MSTU, № 9, pp. 12-21, 2015. 